\title{
PHƯƠNG PHÁP KIỂM ĐỊNH TỌA Độ ĐO BẰNG MÁY TOÀN ĐẠC ĐIỆN TỬ
}

\author{
BÙI ĐĂNG QUANG ${ }^{(1)}$, NGUYẼ̃N VĂN SÁNG ${ }^{(2)}$, \\ NGUYẼ̃N TH! THANH HƯONG ${ }^{(3)}$ \\ ${ }^{(1)}$ Cuc Đo đạc, Bản đồ và Thông tin địa lý Việt Nam \\ ${ }^{(2)}$ Truoòng Đại học Mỏ - Địa chất \\ (3) Viện Khoa học Đo đạc và Bản đồ
}

\section{Tóm tắt:}

Trước mỗi công trình hoặc định kỳ máy toàn đạc điện tủ phải được kiểm định để đánh giá xem máy có đáp úng yêu cầu của công trình hay không. Một trong nhũng kết quả đo của máy toàn đạc điện tư là tọa độ. Ỏ Việt Nam chura có công trình nghiên cúu nào về kiểm định kết quả đo này. Bài báo trình bày quy trình kiểm định tọa độ đo bằng máy toàn đạc điện tủ. Quy trình kiểm định được nghiên cứu chi tiết tù̀ việc bố trí bãi kiểm định, trình tụ đo đạc và các buớc tính toán. Quy trình kiểm định này phù hợp với tiêu chuẩn của quốc tế. Thưc nghiệm kiểm định tọa độ được thực hiện đối vớ máy toàn đạc điện tử Sokkia SET 520K. Kết quả thực nghiệm cho thấy độ lệch chuẩn kiểm định tọa độ của máy đạt $\pm 0,8 \mathrm{~mm}$.

\section{Giới thiệu}

Hiện nay, máy toàn đạc điện tử đã và đang được sử dụng rộng rãi trên thế giới cũng như ở Việt Nam trong thực tế thi công các công trình đo đạc. Trước khi đưa vào sử dụng hoặc định kỳ máy toàn đạc điện tử phải được kiểm định để đánh giá xem máy có đáp ứng yêu cầu của cata$\log$ để đảm bảo chất lượng công trình không. Trước đây, máy toàn đạc điện tử không thuộc danh mục phương tiện đo nhóm 2 , do vậy chưa có quy định chính thức nào về kiểm định, hiệu chuẩn máy toàn đạc điện tử. Các đơn vị kiểm định máy chỉ dựa vào một số kết quả nghiên cứu, thực nghiệm để tự xây dựng các quy trình kiểm định, hiệu chuẩn nội bộ và cũng chưa được co quan quản lý nhà nước có thẩm quyền công nhận. Theo Thông tư 07/2019/TT-BKHCN ngày 26 tháng 7 năm 2019 thì máy toàn đạc điện tử thuộc danh mục phương tiện đo nhóm 2 phải kiểm định theo yêu cầu kỹ thuật đo lường do cơ quan quản lý nhà nước về đo lường có thẩm quyền quy định áp dụng.

Hiện nay, Quy trình Kiểm định máy toàn đạc điện tử đã được Tổng cục Tiêu chuẩn Đo lường
Chất lượng xây dựng và đưa vào áp dụng. Do thông tin về độ chính xác trong catalog của các máy toàn đạc điện tử chủ yếu đưa ra cho giá trị góc và cạnh, vì vậy Quy trình kiểm định hiện nay được xây dựng cũng phải bám theo các thông số trong catalog của máy để sau quá trình kiểm định có căn cứ kết luận máy có đạt yêu cầu về chất lượng hay không. Quy trình đã được xây dựng dựa trên một số phương pháp và kỹ thuật của tổ chức tiêu chuẩn quốc tế về kiểm định máy toàn đạc điện tử, cụ thể là: ISO 17123-3 năm 2001 đã đưa ra các quy định về kiểm định bộ phận đo góc [4]; ISO 17123-4 năm 2012 đã đưa ra các quy định về kiểm định bộ phận đo khoảng cách [5].

Ở Việt Nam, các nghiên cứu về kiểm định máy toàn đạc điện tử được thực hiện từ những năm 2000. Ví dụ, trong tài liệu [8], tác giả đã nghiên cứu sự phụ thuộc giữa hằng số cộng và hằng số nhân máy đo khoảng cách điện tử. Từ đó, đưa ra quy định về kiểm định máy đo khoảng cách điện tử. Trong tài liệu [2] đã đưa ra cách phương pháp để kiểm định hằng số cộng và hằng số nhân của máy toàn đạc điện tử. Tài liệu này cũng đưa ra các quy định về kiểm nghiệm bộ

Ngày nhận bài: 05/3/2021, ngày chuyển phản biện: 09/3/2021, ngày chấp nhận phản biện: 15/3/2021, ngày chấp nhận đăng: 18/3/2021 
phận đo góc, nhưng chủ yếu cho máy kinh vĩ. Trong tài liệu [3] đã đưa ra các quy định về kiểm định máy kinh vĩ đo góc chính xác. Trong tài liệu [1], các tác giả đã đưa ra cấu tạo và nguyên lý hoạt động của máy đo xa điện tử nhưng chưa đưa ra phương pháp kiểm định. Trong [10], các tác giả đã nghiên cứu phương pháp hiệu chuẩn máy đo xa quang - điện tử, tính toán lựa chọn đường chuẩn đề kiểm định máy đo xa điện tử. Tuy nhiên, cho đến nay chưa có công trình nghiên cứu nào về kiểm định tọa độ đo bằng máy toàn đạc điện tử.

Hiện nay, khi đo đạc các công trình chủ yếu quy định độ chính xác về tọa độ. Do vậy về lâu dài, các máy toàn đạc điện tử sẽ có thông tin đầy đủ về độ chính xác đo góc, cạnh và tọa độ. Vì vậy, để đáp ứng được yêu cầu về kiểm định tọa độ cho máy toàn đạc điện tử (khi catalog của các máy có nhũng thông số độ chính xác về tọa độ) cũng như để hội nhập với thế giới thì Việt Nam cần có những nghiên cứu về quy định kiểm định, hiệu chuẩn máy toàn đạc điện tử tương ứng, phù hợp với thế giới, có xem xét đến các đặc điểm của Việt Nam. Do đó, bài báo này tập trung nghiên cứu về quy trình kiểm định tọa độ đo bằng máy toàn đạc điện tử ở Việt Nam và trình bày kết quả thực nghiệm kiểm định tọa độ.

\section{Quy trình kiểm định tọa độ đo bằng máy toàn đạc điện tử}

\subsection{Phương pháp bố trí bãi kiểm định tọa đọ}

Để kiểm định tọa độ, cần bố trí bãi kiểm định như sau [6,7]: Ba mục tiêu (gương) (T1, T2, T3) phải được đặt ở ba góc của tam giác (Hình 1). Các mục tiêu cần được cố định chắc chắn trên mặt đất. Khoảng cách giữa các điểm mục tiêu phải khác nhau và ít nhất một khoảng cách phải dài hơn khoảng cách trung bình (ví dụ: $60 \mathrm{~m}$ ) theo nhiệm vụ đo đạc dự định. Chiều cao của chúng phải khác nhau.

Ba trạm máy toàn đạc điện tử $(\mathrm{S} 1, \mathrm{~S} 2, \mathrm{~S} 3)$ phải được đặt gần trên mỗi cạnh tam giác, cách mỗi điểm mục tiêu khoảng $5 \mathrm{~m}$ đến $10 \mathrm{~m}$ (Hình $1)$.

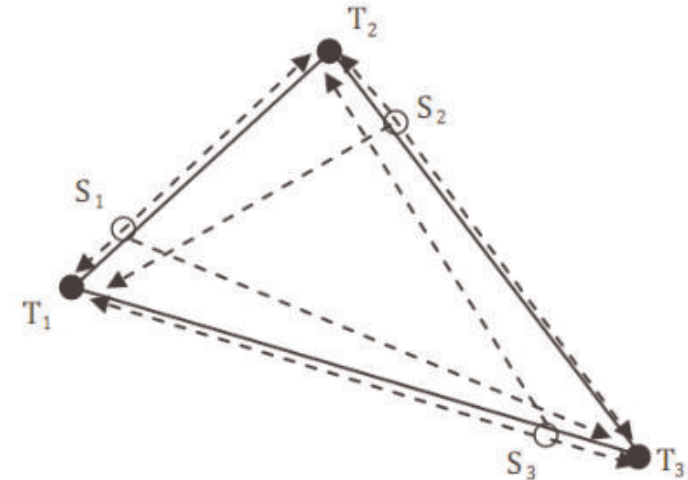

Hình 1: Bố trí bãi kiểm định tọa độ

\subsection{Quy trình đo đạc trong kiểm định tọa độ}

Để kiểm định tọa độ, chúng ta cần đo đạc theo các bước sau $[6,7]$ :

1- Đặt máy tại điểm $\mathrm{S}_{1}$, định tâm, cần bằng máy cẩn thận. Ở vị trí bàn độ trái, lần lượt đo tọa độ đến các gương $\mathrm{T}_{1}, \mathrm{~T}_{2}, \mathrm{~T}_{3}$, các kết quả được ghi vào các ô tương ứng trong Bảng 1 , được 1 lần đo.

2- Đảo ống ngắm, ở vị trí bàn độ phải, lần lượt đo tọa độ đến các gương $\mathrm{T}_{1}, \mathrm{~T}_{2}, \mathrm{~T}_{3}$, được lần đo thứ 2

3- Lặp lại thao tác trên một lần nữa, được lần đo thứ 3 và 4 .

4- Chuyển máy đến điểm $\mathrm{S}_{2}$ và $\mathrm{S}_{3}$ cũng thao tác tương tự như ở $\mathrm{S}_{1}$.

Các tọa độ trạm máy được giả định và định hướng tùy ý đến một trong các gương. Các cấu hình này không nên thay đổi trong khi đo bốn lần đo tại cùng một điểm trạm máy. Trình tự và kết quả các phép đo được thể hiện trong Bảng 1.

\subsection{Tính toán trong kiểm định tọa độ}

Việc tính toán trong kiểm định tọa độ được thực hiện theo trình tự sau $[6,7]$ :

- Từ tọa độ của $T_{1}$ và $T_{2}$ tính ra khoảng cách ngang giữa điểm $\mathrm{T}_{1}$ và $\mathrm{T}_{2}$ theo công thức:

$$
l_{1,2}=\sqrt{\left(x_{2}-x_{1}\right)^{2}+\left(y_{2}-y_{1}\right)^{2}}
$$

- Từ tọa độ của $T_{2}$ và $T_{3}$ tính ra khoảng cách 


\section{Nghiên cứu}

Bảng 1: Trình tụ các phép đo và ghi tọa độ

\begin{tabular}{|c|c|c|c|c|c|c|}
\hline No & $\begin{array}{c}\text { Trạm máy } \\
i\end{array}$ & $\begin{array}{c}\text { Mục tiêu } \\
\mathrm{j}\end{array}$ & $\begin{array}{c}\text { Bộ đo } \\
\mathrm{k}\end{array}$ & $\begin{array}{c}\text { Vị trí } \\
\text { ông kính }\end{array}$ & $\mathrm{x}_{\mathrm{i}, \mathrm{j}, \mathrm{k}}(\mathrm{m})$ & $\mathrm{y}_{\mathrm{i}, \mathrm{j}, \mathrm{k}}(\mathrm{m})$ \\
\hline 1 & \multirow{12}{*}{1} & 1 & \multirow{3}{*}{1} & \multirow{3}{*}{ I } & $\mathrm{x}_{1,1,1}$ & $\mathrm{y}_{1,1,1}$ \\
\hline 2 & & 2 & & & $\mathrm{x}_{1,2,1}$ & $\mathrm{y}_{1,2,1}$ \\
\hline 3 & & 3 & & & $\mathrm{x}_{1,3,1}$ & $\mathrm{y}_{1,3,1}$ \\
\hline 4 & & 1 & \multirow{3}{*}{2} & \multirow{3}{*}{ II } & $\mathrm{x}_{1,1,2}$ & $\mathrm{y}_{1,1,2}$ \\
\hline 5 & & 2 & & & $\mathrm{x}_{1,2,2}$ & $\mathrm{y}_{1,2,2}$ \\
\hline 6 & & 3 & & & $\mathrm{x}_{1,3,2}$ & $\mathrm{y}_{1,3,2}$ \\
\hline 7 & & 1 & \multirow{3}{*}{3} & \multirow{3}{*}{ I } & $\mathrm{x}_{1,1,3}$ & $\mathrm{y}_{1,1,3}$ \\
\hline 8 & & 2 & & & $\mathrm{x}_{1,2,3}$ & $\mathrm{y}_{1,2,3}$ \\
\hline 9 & & 3 & & & $\mathrm{x}_{1,3,3}$ & $\mathrm{y}_{1,3,3}$ \\
\hline 10 & & 1 & \multirow{3}{*}{4} & \multirow{3}{*}{ II } & $\mathrm{x}_{1,1,4}$ & $\mathrm{y}_{1,1,4}$ \\
\hline 11 & & 2 & & & $\mathrm{x}_{1,2,4}$ & $\mathrm{y}_{1,2,4}$ \\
\hline 12 & & 3 & & & $\mathrm{x}_{1,3,4}$ & $\mathrm{y}_{1,3,4}$ \\
\hline 13 & 2 & 1 & 1 & I & $\mathrm{x}_{2,1,1}$ & $\mathrm{y}_{2,1,1}$ \\
\hline$\ldots$ & $\ldots$ & $\ldots$ & & & & \\
\hline 34 & & 1 & & & $\mathrm{x}_{3,1,4}$ & $\mathrm{y}_{3,1,4}$ \\
\hline 35 & 3 & 2 & 4 & II & $\mathrm{x}_{3,2,4}$ & $\mathrm{y}_{3,2,4}$ \\
\hline 36 & & & & & & \\
\hline
\end{tabular}

ngang giữa điểm $\mathrm{T}_{2}$ và $\mathrm{T}_{3}$ theo công thức:

$$
l_{2,3}=\sqrt{\left(x_{3}-x_{2}\right)^{2}+\left(y_{3}-y_{2}\right)^{2}}
$$

- Từ tọa độ của $T_{1}$ và $T_{3}$ tính ra khoảng cách ngang giữa điểm $T_{1}$ và $T_{3}$ theo công thức:

$$
l_{1,3}=\sqrt{\left(x_{3}-x_{1}\right)^{2}+\left(y_{3}-y_{1}\right)^{2}}
$$

Mỗi khoảng cách ngang sẽ tính được 12 giá trị. Tính trung bình của các cạnh theo công thức:

$$
L_{1,2}=\frac{1}{12} \sum_{j=1}^{12} l_{1,2, j}
$$

Tương tự với $L_{2,3}$ và $L_{1,3}$.

- Các tọa độ của mô hình toán học của tam giác $M_{1} M_{2} M_{3}$ được xác định như sau:

Điểm $\mathrm{M}_{1}=(0,0)$ trùng với gốc tọa độ. Đường thẳng từ $\mathrm{M}_{1}$ đến $\mathrm{M}_{2}$ được chọn làm trục $\mathrm{x}$. Đường vuông góc là trục $\mathrm{y}$ (Hình 2).

Điểm $\mathrm{M}_{2}$ có tọa độ là $\left(\mathrm{L}_{1,2}, 0\right)$

Tọa độ của điểm $\mathrm{M}_{3}\left(\mathrm{X}_{3}, \mathrm{Y}_{3}\right)$ sẽ là:

$$
X_{3}=\frac{-L_{2,8}^{2}+L_{1,8}^{2}+L_{1,2}^{2}}{2 L_{1,2}}
$$

$$
Y_{3}=\sqrt{L_{1,3}^{2}-\left[\frac{-L_{2,8}^{2}+L_{1,3}^{2}+L_{1,2}^{2}}{2 L_{1,2}}\right]^{2}}=\sqrt{L_{1,3}^{2}-X_{3}^{2}}
$$

Các tọa độ trọng tâm của mô hình toán học, (Xg, Yg):

$$
\left(X_{g}, Y_{g}\right)=\left(\frac{\sum_{j=1}^{8} x_{j}}{3}, \frac{\sum_{j=1}^{s} Y_{j}}{3}\right)
$$

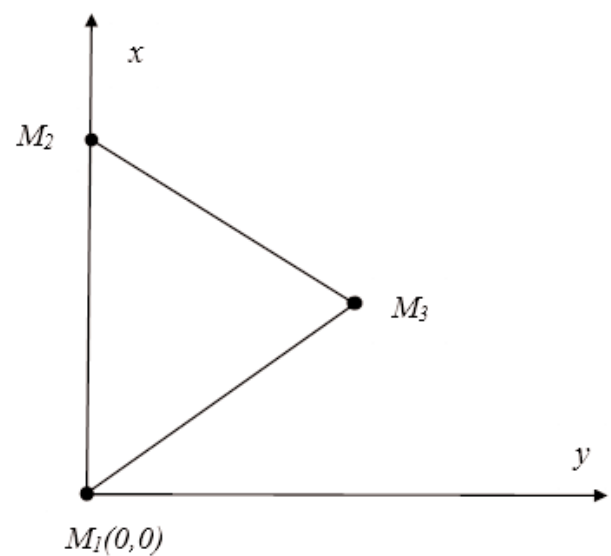

Hình 2: Mô hình toán của tam giác

- Tọa độ trọng tâm đo của tam giác thu được tại mỗi trạm máy $i,\left(\mathrm{x}_{\mathrm{g}, \mathrm{i}}, \mathrm{y}_{\mathrm{g}, \mathrm{i}}\right)$ : 


$$
\left(x_{g, i}, y_{g, i}\right)=\left(\frac{\sum_{j=1}^{3} \sum_{k=1}^{4} x_{i, j, k}}{12}, \frac{\sum_{j=1}^{j} \sum_{k=1}^{4} y_{i, j, k}}{12}\right)
$$

- Tính độ lệch tọa độ trọng tâm của mô hình tam giác và tọa độ đo:

$$
\Delta x_{g, i}=x_{g, i}-X_{g} ; \quad \Delta y_{g, i}=y_{g, i}-Y_{g} .
$$

- Hiệu chỉnh tọa độ để trùng khớp trọng tâm của mô hình toán học lên trọng tâm của tam giác đo. Các tọa độ của mô hình toán học $\left(\mathrm{X}_{\mathrm{t}, \mathrm{i}, \mathrm{k}}\right.$, $\mathrm{Y}_{\mathrm{t}, \mathrm{i}, \mathrm{k}}$ ) sau khi hiệu chỉnh được tính như sau:

$$
\begin{aligned}
& X_{t, i, j, k}=X_{j}+\Delta x_{g, i} ; Y_{t, i, j, k}=Y_{j}+\Delta y_{g, i} ; \\
& i=1,2,3 ; j=1,2,3 ; k=1,2,3,4
\end{aligned}
$$

- Xoay mô hình toán học xung quanh trọng tâm để giảm thiểu các phần dư của tọa độ đỉnh giữa mô hình toán học và các tam giác đo tương ứng.

Góc xoay $\Theta_{i, k}$ được tính theo công thức:

$$
\theta_{i, k}=\tan ^{-1}\left(\frac{q_{i, k}}{p_{i, k}}\right) ; i=1,2,3 ; k=1,2,3,4,
$$

trong đó:

$q_{i, k}=\frac{\sum_{j=1}^{z}\left(\left(x_{t, i, k}-x_{g, i}\right) \times\left(y_{i, j, k}-y_{g, i}\right)-\left(y_{t, i j, k}-y_{g, i}\right) \times\left(x_{i, j, k}-x_{g, i}\right)\right)}{\sum_{j=1}^{z}\left(\left(x_{t, i, j, k}-x_{g, i}\right)^{2}+\left(y_{t, i, j, k}-y_{g, i}\right)^{2}\right)}$

$$
p_{i, k}=\frac{\sum_{j=1}^{3}\left(\left(x_{t i, j, k}-x_{g, i}\right) \times\left(x_{i, j, k}-x_{g, i}\right)-\left(y_{t, i, k}-y_{g, i}\right) \times\left(y_{i, j, k}-y_{g, i}\right)\right)}{\sum_{j=1}^{3}\left(\left(x_{t, i, j, k}-x_{g, j}\right)^{2}+\left(y_{t, i, j, k}-y_{g, i}\right)^{2}\right)}
$$

Tọa độ các đỉnh của mô hình toán học $\left(X_{\mathrm{m}, \mathrm{i}, \mathrm{j}, \mathrm{k}}, Y_{\mathrm{m}, \mathrm{i}, \mathrm{j}, \mathrm{k}}\right)$ sau khi quay được tính theo công thức:

$$
\begin{gathered}
X_{m, i, j, k}=x_{g, i}+\cos \theta_{i, k} \times\left(X_{t, i, j, k}-x_{g, i}\right)- \\
-\sin \theta_{i, k} \times\left(Y_{t, i, j, k}-y_{g, i}\right) ; \\
Y_{m, i, j, k}=y_{g, i}+\sin \theta_{i, k} \times\left(X_{t, i, j, k}-x_{g, i}\right)- \\
-\cos \theta_{i, k} \times\left(Y_{t, i, j, k}-y_{g, i}\right),
\end{gathered}
$$

vói $i=1,2,3 ; j=1,2,3 ; k=1,2,3,4$.

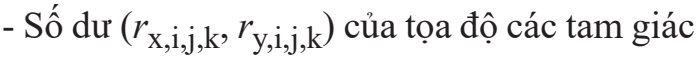
đo được so với mô hình toán học đã xoay là:

$$
\begin{aligned}
& r_{x, i, j, k}=x_{i, j, k}-X_{m, i, j, k} ; \\
& r_{y, i, j, k}=y_{i, j, k}-Y_{m, i, j, k} .
\end{aligned}
$$

- Tổng bình phương của phần dư là:

$$
\sum r_{x y}^{2}=\sum_{i=1}^{3} \sum_{j=1}^{3} \sum_{k=1}^{4}\left(r_{x, i, j, k}^{2}+r_{y, i, j, k}^{2}\right) \text {. }
$$

- Độ lệch chuẩn kiểm định tọa độ $\mathrm{x}, \mathrm{y}$ là:

$$
S_{X Y}= \pm \sqrt{\frac{\sum r_{x y}^{2}}{t}},
$$

trong đó: t là số bậc tự do.

\section{Thực nghiệm kiểm định tọa độ đo bằng máy toàn đạc điện tử}

\subsection{Thưc nghiệm bố trí bãi kiểm định tọa độ}

Bãi kiểm định tọa độ được bố trí tại Khu đô thị Resco - Cổ Nhuế 2 - Bắc Từ Liêm - Hà Nội. Ba mục tiêu (gương) (T1, T2, T3) được đặt ở các góc của tam giác (Hình 3).

Các mục tiêu được cố định chắc chắn trên mặt đất. Khoảng cách mục tiêu $\mathrm{T} 1$ đến $\mathrm{T} 2$ là $92,7 \mathrm{~m}$, từ $\mathrm{T} 2$ đến $\mathrm{T} 3$ là $66,5 \mathrm{~m}$, từ $\mathrm{T} 1$ đến $\mathrm{T} 3$ là $71 \mathrm{~m}$. Chiều cao của các điểm khác nhau. Ba trạm máy toàn đạc (S1, S2, S3) được đặt trên các cạnh của tam giác, cách mỗi điểm mục tiêu khoảng $5 \mathrm{~m}$ đến $10 \mathrm{~m}$. Khi bố trí, khoảng cách giữa các điểm được đo sơ bộ bằng máy toàn đạc điện tử. Khi tính toán, khoảng cách giữa các gương được tính từ tọa độ đo được.

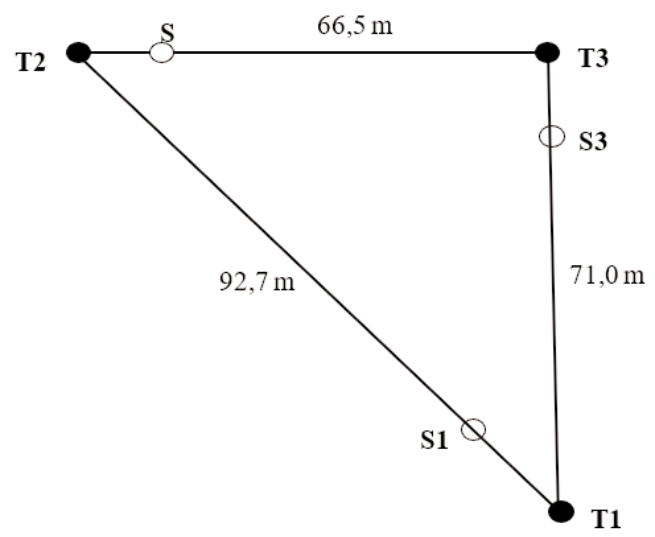

Hình 3: Bãi kiểm định tọa độ thực nghiệm 


\subsection{Kết quả đo kiểm định tọa độ}

Đo thực nghiệm kiểm định tọa độ được thực hiện ngày 23/12/2020, trong điều kiện thời tiết râm mát, nhiệt độ là $20^{\circ} \mathrm{C}$, áp suất là $1012 \mathrm{mbar}$, máy kiểm định là máy Sokkia SET 520K, số máy 173918. Độ chính xác đo góc là 5", độ chính xác đo cạnh là $2+2 \mathrm{ppm}$.D.

Khi đo tại điểm $\mathrm{S}$ 1, định hướng về điểm $\mathrm{T} 2$, lấy tọa độ điểm S1 $(x=100,000 \mathrm{~m}, \mathrm{y}=200,000$ $\mathrm{m})$. Khi đo tại điểm $\mathrm{S} 2$, định hướng về điểm $\mathrm{T} 1$, lấy tọa độ điểm S1 $(\mathrm{x}=200,000 \mathrm{~m}, \mathrm{y}=300,000$ $\mathrm{m})$. Khi đo tại điểm $\mathrm{S} 3$, định hướng về điểm $\mathrm{T} 1$, lấy tọa độ điểm $\mathrm{S} 1(\mathrm{x}=300,000 \mathrm{~m}, \mathrm{y}=400,000$ $\mathrm{m})$. Kết quả đo được trình bày trong Bảng 2 .

\subsection{Kết quả tính toán kiểm định tọa độ}

+) Tính khoảng cách giữa các gương theo tọa độ đo được:

Khoảng cách giữa các gương được tính theo công thức (1), (2) và (3). Khoảng cách trung bình được tính theo công thức (4). Kết quả tính toán được trình bày trong Bảng 3 .

Bảng 3: Kết quả tính khoảng cách giũa các guoong

\begin{tabular}{|c|c|c|c|}
\hline STT & $l_{l, 2}(m)$ & $l_{2,3}(m)$ & $l_{l, 3}(m)$ \\
\hline 1 & 92,7420 & 66,5659 & 71,0189 \\
\hline 2 & 92,7410 & 66,5646 & 71,0188 \\
\hline 3 & 92,7420 & 66,5646 & 71,0188 \\
\hline 4 & 92,7420 & 66,5638 & 71,0188 \\
\hline 5 & 92,7426 & 66,5640 & 71,0202 \\
\hline 6 & 92,7425 & 66,5638 & 71,0201 \\
\hline 7 & 92,7424 & 66,5638 & 71,0208 \\
\hline 8 & 92,7425 & 66,5644 & 71,0187 \\
\hline 9 & 92,7449 & 66,5643 & 71,0180 \\
\hline 10 & 92,7434 & 66,5642 & 71,0180 \\
\hline 11 & 92,7448 & 66,5642 & 71,0180 \\
\hline 12 & 92,7420 & 66,5644 & 71,0180 \\
\hline TB (L) & $\mathbf{9 2 , 7 4 2 7}$ & $\mathbf{6 6 , 5 6 4 3}$ & $\mathbf{7 1 , 0 1 9 0}$ \\
\hline
\end{tabular}

+) Tính tọa độ của mô hình toán học của tam giác $M_{1} M_{2} M_{3}$ và tọa độ trọng tâm mô hình $G$ :

Tọa độ của các đỉnh tam giác $M_{1} M_{2} M_{3}$ trong mô hình toán được tính theo công thức (5), (6). Tọa độ trọng tâm $\mathrm{G}$ của mô hình được tính theo công thức (7). Kết quả được trình bày trên Bảng 4.

\section{Bảng 4: Tọa độ trong mô hình toán học}

\begin{tabular}{|c|r|r|}
\hline Điểm & \multicolumn{1}{|c|}{$X(\mathrm{~m})$} & \multicolumn{1}{c|}{$Y(\mathrm{~m})$} \\
\hline $\mathrm{M} 1$ & 0,0000 & 0,0000 \\
\hline $\mathrm{M} 2$ & 92,7427 & 0,0000 \\
\hline $\mathrm{M} 3$ & 49,6755 & 50,7546 \\
\hline G & $\mathbf{4 7 , 4 7 2 7}$ & $\mathbf{1 6 , 9 1 8 2}$ \\
\hline
\end{tabular}

+) Tính tọa độ trọng tâm của tam giác thu được tại mỗi trạm máy:

Từ tọa độ đo được của các trạm máy, tọa độ trọng tâm của tam giác được tính theo công thức (8). Kết quả như sau:

Tọa độ trọng tâm g tại trạm máy 1 :

$$
\mathrm{x}=141,1013 \mathrm{~m} ; \mathrm{y}=216,9182 \mathrm{~m} .
$$

Tọa độ trọng tâm g tại trạm máy 2 :

$$
\mathrm{x}=239,0547 \mathrm{~m} ; \mathrm{y}=286,3568 \mathrm{~m} \text {. }
$$

Tọa độ trọng tâm g tại trạm máy 3:

$$
\mathrm{x}=319,7118 \mathrm{~m} ; \mathrm{y}=422,0400 \mathrm{~m} \text {. }
$$

+) Độ lệch tọa độ trọng tâm giữa mô hình tam giác và tọa độ đo được tính theo công thức (9). Kết quả được trình bày trong Bảng 5 .

Bảng 5: Độ lệch tọa độ trọng tâm

\begin{tabular}{|c|c|c|}
\hline Trạm máy & $\Delta \mathrm{x}(\mathrm{m})$ & $\Delta \mathrm{y}(\mathrm{m})$ \\
\hline 1 & 93,6285 & 200,0000 \\
\hline 2 & 191,5819 & 269,4385 \\
\hline 3 & 272,2391 & 405,1218 \\
\hline
\end{tabular}

+) Hiệu chỉnh tọa độ để trùng khớp trọng tâm của mô hình toán học lên trọng tâm của tam giác đo theo công thức (10). Kết quả được trình bày trong Bảng 6.

+) Tính tọa độ sau khi xoay theo công thức (14) và (15). Kết quả được trình bày trong Bảng 7.

+) Tính số dư $\left(r_{\mathrm{x}, \mathrm{i}, \mathrm{j}, \mathrm{k}}, r_{\mathrm{y}, \mathrm{i}, \mathrm{j}, \mathrm{k}}\right)$ của tọa độ các tam giác đo được so với mô hình toán học đã 
Bảng 2: Kết quả đo thực nghiệm kiểm định tọa độ

\begin{tabular}{|c|c|c|c|c|c|c|}
\hline No & $\begin{array}{l}\text { Trạm } \\
\text { máy }\end{array}$ & $\begin{array}{l}\text { Mục } \\
\text { tiêul }\end{array}$ & $\begin{array}{l}\text { Bộ } \\
\text { do }\end{array}$ & $\begin{array}{l}\text { Bàn } \\
\text { độ }\end{array}$ & $x(m)$ & $\mathrm{y}(\mathrm{m})$ \\
\hline 1 & \multirow{10}{*}{1} & 1 & \multirow{3}{*}{1} & \multirow{3}{*}{$\mathrm{T}$} & 93,629 & 200,000 \\
\hline 2 & & 2 & & & 186,371 & 200,000 \\
\hline 3 & & 3 & & & 143,303 & 250,756 \\
\hline 4 & & 1 & \multirow{3}{*}{2} & \multirow{3}{*}{$\mathrm{P}$} & 93,629 & 200,000 \\
\hline 5 & & 2 & & & 186,370 & 199,999 \\
\hline 6 & & 3 & & & 143,304 & 250,755 \\
\hline$\ldots$ & & $\ldots$ & $\ldots$ & $\ldots$ & & \\
\hline 10 & & 1 & \multirow{3}{*}{4} & \multirow{3}{*}{$\mathrm{P}$} & 93,629 & 200,000 \\
\hline 11 & & 2 & & & 186,371 & 200,001 \\
\hline 12 & & 3 & & & 143,304 & 250,755 \\
\hline$\ldots$ & $\ldots$ & $\ldots$ & $\ldots$ & $\ldots$ & $\ldots$ & $\ldots$ \\
\hline 13 & \multirow{3}{*}{2} & 1 & \multirow{3}{*}{1} & \multirow{3}{*}{$\mathrm{T}$} & 287,570 & 300,000 \\
\hline 14 & & 2 & & & 195,043 & 306,320 \\
\hline 15 & & 3 & & & 234,550 & 252,748 \\
\hline$\ldots$ & $\ldots$ & $\ldots$ & $\ldots$ & $\ldots$ & $\ldots$ & $\ldots$ \\
\hline 34 & \multirow{3}{*}{3} & 1 & \multirow{3}{*}{4} & \multirow{3}{*}{$\mathrm{P}$} & 365,034 & 400,000 \\
\hline 35 & & 2 & & & 300,087 & 466,204 \\
\hline 36 & & 3 & & & 294,016 & 399,917 \\
\hline
\end{tabular}

Bảng 6: Tọa độ sau khi hiệu chỉnh

\begin{tabular}{|c|c|c|c|c|c|c|c|}
\hline Trạm máy & Bộ đo & \multicolumn{2}{|c|}{ Gương 1} & \multicolumn{2}{c|}{ Gương 2} & \multicolumn{2}{c|}{ Gương 3} \\
\hline$i$ & $k$ & $X_{t, i, 1, k}$ & $\boldsymbol{Y}_{t, i, 1, k}$ & $\boldsymbol{X}_{\boldsymbol{t}, i, 2, k}$ & $\boldsymbol{Y}_{t, i, 2, k}$ & $\boldsymbol{X}_{\boldsymbol{t}, i, 3, k}$ & $\boldsymbol{Y}_{t, i, 3, k}$ \\
\hline 1 & 1 & 93,6285 & 200,0000 & 186,3712 & 200,0000 & 143,3041 & 250,7546 \\
\hline 1 & 2 & 93,6285 & 200,0000 & 186,3712 & 200,0000 & 143,3041 & 250,7546 \\
\hline 1 & 3 & 93,6285 & 200,0000 & 186,3712 & 200,0000 & 143,3041 & 250,7546 \\
\hline 1 & 4 & 93,6285 & 200,0000 & 186,3712 & 200,0000 & 143,3041 & 250,7546 \\
\hline 2 & 1 & 191,5819 & 269,4385 & 284,3246 & 269,4385 & 241,2575 & 320,1932 \\
\hline 2 & 2 & 191,5819 & 269,4385 & 284,3246 & 269,4385 & 241,2575 & 320,1932 \\
\hline 2 & 3 & 191,5819 & 269,4385 & 284,3246 & 269,4385 & 241,2575 & 320,1932 \\
\hline 2 & 4 & 191,5819 & 269,4385 & 284,3246 & 269,4385 & 241,2575 & 320,1932 \\
\hline 3 & 1 & 272,2391 & 405,1218 & 364,9818 & 405,1218 & 321,9146 & 455,8764 \\
\hline 3 & 2 & 272,2391 & 405,1218 & 364,9818 & 405,1218 & 321,9146 & 455,8764 \\
\hline 3 & 3 & 272,2391 & 405,1218 & 364,9818 & 405,1218 & 321,9146 & 455,8764 \\
\hline 3 & 4 & 272,2391 & 405,1218 & 364,9818 & 405,1218 & 321,9146 & 455,8764 \\
\hline
\end{tabular}

xoay theo công thức (16). Kết quả được trình bày trong Bảng 8 .

+) Tổng bình phương của phần dư là:

$$
\sum r_{x y}^{2}=\sum_{i=1}^{3} \sum_{j=1}^{3} \sum_{k=1}^{4}\left(r_{x, i, j, k}^{2}+r_{y, i, j, k}^{2}\right)=0,0000315
$$

+) Tính độ lệch chuẩn kiểm định tọa độ $\mathrm{x}, \mathrm{y}$ :

$$
s_{X Y}=\sqrt{\frac{\sum_{x y}^{2}}{t}}=\sqrt{\frac{\sum_{i=1}^{3} \sum_{j=1}^{3} \sum_{k=1}^{4}\left(r_{x, i j, k}^{2}+r_{y, i, k}^{2}\right)}{t}}= \pm 0,0008 \mathrm{~m}
$$

Như vậy, máy có độ lệch chuẩn kiểm định tọa độ là: $\pm 0,0008$ m.

\section{Kết luận}

Bài báo đã trình bày quy trình kiểm định tọa độ đo bằng máy toàn đạc điện tử với đầy đủ các nội dung từ bố trí bãi kiểm định đến trình tự đo đạc và trình tự tính toán. Phương pháp này phù hợp với tiêu chuẩn quốc tế.

Quy trình kiểm định tọa độ đo bằng máy toàn đạc điện tử đã được tiến hành thực nghiệm tại Việt Nam đối với máy Sokkia SET 520K, số máy 173918. Kết quả tính toán kiểm định cho thấy máy có độ lệch chuẩn kiểm định tọa độ là $\pm 0,8 \mathrm{~mm}$. $\mathrm{O}$ 
Bảng 7: Tọa độ sau khi xoay

\begin{tabular}{|c|c|r|r|c|c|c|c|}
\hline Trạm máy & Bộ đo & \multicolumn{2}{|c|}{ Gương 1 } & \multicolumn{2}{c|}{ Gương 2} & \multicolumn{2}{c|}{ Gương 3 } \\
\hline $\mathrm{i}$ & $\mathrm{k}$ & $\mathrm{X}_{\mathrm{m}, \mathrm{i}, 1, \mathrm{k}}$ & $\mathrm{Y}_{\mathrm{m}, \mathrm{i}, 1, \mathrm{k}}$ & $\mathrm{X}_{\mathrm{m}, \mathrm{i}, 2, \mathrm{k}}$ & $\mathrm{Y}_{\mathrm{m}, \mathrm{i}, 2, \mathrm{k}}$ & $\mathrm{X}_{\mathrm{m}, \mathrm{i}, 3, \mathrm{k}}$ & $\mathrm{Y}_{\mathrm{m}, \mathrm{i}, 3, \mathrm{k}}$ \\
\hline 1 & 1 & 93,6286 & 199,9996 & 186,3713 & 200,0003 & 143,3038 & 250,7546 \\
\hline 1 & 2 & 93,6284 & 200,0004 & 186,3710 & 199,9995 & 143,3044 & 250,7546 \\
\hline 1 & 3 & 93,6282 & 200,0009 & 186,3709 & 199,9991 & 143,3047 & 250,7545 \\
\hline 1 & 4 & 93,6287 & 199,9995 & 186,3713 & 200,0004 & 143,3038 & 250,7546 \\
\hline 2 & 1 & 287,5700 & 300,0002 & 195,0430 & 306,3209 & 234,5510 & 252,7491 \\
\hline 2 & 2 & 287,5698 & 300,0013 & 195,0426 & 306,3200 & 234,5517 & 252,7490 \\
\hline 2 & 3 & 287,5696 & 300,0017 & 195,0424 & 306,3196 & 234,5519 & 252,7490 \\
\hline 2 & 4 & 287,5698 & 300,0011 & 195,0426 & 306,3201 & 234,5516 & 252,7490 \\
\hline 3 & 1 & 365,0337 & 399,9988 & 300,0870 & 466,2040 & 294,0148 & 399,9172 \\
\hline 3 & 2 & 365,0344 & 400,0002 & 300,0857 & 466,2034 & 294,0155 & 399,9164 \\
\hline 3 & 3 & 365,0344 & 400,0001 & 300,0857 & 466,2034 & 294,0155 & 399,9164 \\
\hline 3 & 4 & 365,0340 & 399,9995 & 300,0863 & 466,2037 & 294,0151 & 399,9168 \\
\hline
\end{tabular}

Bảng 8: Kết quả tính số du

\begin{tabular}{|c|r|r|r|r|r|r|r|}
\hline Trạm máy & Bộ đo & \multicolumn{2}{|c|}{ Gương 1 } & \multicolumn{2}{c|}{ Gương 2 } & \multicolumn{2}{c|}{ Gương 3 } \\
\hline $\mathrm{i}$ & $\mathrm{k}$ & $\mathrm{r}_{\mathrm{x}, \mathrm{i}, 1, \mathrm{k}}(\mathrm{m})$ & \multicolumn{1}{c|}{$\mathrm{r}_{\mathrm{y}, \mathrm{i}, 1, \mathrm{k}}(\mathrm{m})$} & $\mathrm{r}_{\mathrm{x}, \mathrm{i}, 2, \mathrm{k}}(\mathrm{m})$ & $\mathrm{r}_{\mathrm{y}, \mathrm{i}, 2, \mathrm{k}}(\mathrm{m})$ & $\mathrm{r}_{\mathrm{x}, \mathrm{i}, 3, \mathrm{k}}(\mathrm{m})$ & $\mathrm{r}_{\mathrm{y}, \mathrm{i}, 3, \mathrm{k}}(\mathrm{m})$ \\
\hline 1 & 1 & 0,0004 & 0,0004 & $-0,0003$ & $-0,0003$ & $-0,0008$ & 0,0014 \\
\hline 1 & 2 & 0,0006 & $-0,0004$ & $-0,0010$ & $-0,0005$ & $-0,0004$ & 0,0004 \\
\hline 1 & 3 & 0,0008 & $-0,0009$ & 0,0001 & $-0,0011$ & 0,0003 & $-0,0005$ \\
\hline 1 & 4 & 0,0003 & 0,0005 & $-0,0003$ & 0,0006 & 0,0002 & 0,0004 \\
\hline 2 & 1 & 0,0000 & $-0,0002$ & 0,0000 & $-0,0009$ & $-0,0010$ & $-0,0011$ \\
\hline 2 & 2 & 0,0002 & 0,0007 & 0,0004 & 0,0000 & $-0,0007$ & 0,0000 \\
\hline 2 & 3 & 0,0004 & 0,0013 & 0,0006 & 0,0004 & $-0,0009$ & 0,0000 \\
\hline 2 & 4 & 0,0002 & $-0,0001$ & 0,0004 & $-0,0001$ & 0,0004 & 0,0000 \\
\hline 3 & 1 & 0,0003 & $-0,0018$ & $-0,0010$ & 0,0000 & 0,0012 & $-0,0002$ \\
\hline 3 & 2 & $-0,0004$ & $-0,0002$ & $-0,0007$ & 0,0006 & 0,0005 & 0,0006 \\
\hline 3 & 3 & $-0,0004$ & $-0,0011$ & $-0,0017$ & 0,0006 & 0,0005 & 0,0006 \\
\hline 3 & 4 & 0,0000 & 0,0005 & 0,0007 & 0,0003 & 0,0009 & 0,0002 \\
\hline
\end{tabular}

\section{Tài liệu tham khảo}

[1]. Đào Quang Hiếu, Ngô Văn Hợi, 1997. Úng dụng kỹ thuật điện tử trong trắc địa. Bài giảng dành cho học viên cao học. Trường Đại học Mỏ - Địa chất, Hà Nội.

[2]. Đỗ Ngọc Đường, 2000. Xây dựng lưới trắc địa. Trường Đại học Mỏ - Địa chất. Hà Nội.

[3]. Đỗ Ngọc Đường, Đặng Nam Chinh, 2000. Hướng dẫn thực tập Trắc địa cao cấp. Trường đại học Mỏ - Địa chất. Hà Nội.

[4]. International Organization for Standardization, 2001. International Standard ISO 17123-3. Optics and optical instruments Field procedures for testing geodetic and surveying instruments - Part 3: Theodolites.

[5]. International Organization for
Standardization, 2012. International Standard ISO 17123-4. Optics and optical instruments Field procedures for testing geodetic and surveying instruments - Part 4: Electro-optical distance meters (EDM measurements to reflectors).

[6]. International Organization for Standardization, 2012. International Standard ISO 17123-5. Optics and optical instruments Field procedures for testing geodetic and surveying instruments - Part 5: Total stations.

[7]. International Organization for Standardization, 2018. International Standard ISO 17123-5. Optics and optical instruments Field procedures for testing geodetic and surveying instruments - Part 5: Total stations.

[8]. Phạm Doãn Mậu, 2001. Nghiên cứu sự phụ thuộc giữa hằng số cộng và hằng số nhân 
máy đo khoảng cách điện tử. Luận văn thạc sỹ kỹ thuật. Trường Đại học Mỏ - Địa chất, Hà Nội.

[9]. Phạm Hoàng Lân, Đặng Nam Chinh, Dương Vân Phong, Vũ Văn Trí, 2017. Trắc địa cao cấp đại cương. Nhà xuất bản giao thông vận tải. Hà Nội.
[10]. Tống Công Dũng, Vũ Khánh Xuân, Bùi Quốc Thụ, Nguyễn Văn Vinh, 2013. Nghiên cứu xây dựng chuẩn đo lường để hiệu chuẩn máy đo xa quang - điện tử. Tạp chí Khoa học và Kỹ thuật số 158, tr 51-59, ISSN: 1859-0209. Học viện kỹ thuật quân sự. Hà Nội.O

\section{Summary}

Test procedure of the coordinates measured by the electronic total station

Bui Dang Quang, Department of Survey, Mapping and Geographic Information Vietnam

Nguyen Van Sang, Hanoi University of Mining and Geology

Nguyen Thi Thanh Huong, Vietnam Institute of Geodesy and Cartography

Before each project or periodically the electronic total station must be test to assess whether it responses the requirements of the project. One of the measurements of the total station is the coordinates. In Vietnam, there is no research work on test procedure of these measurement results. This paper presents the test procedure of the coordinates measured by the electronic total station. The test procedure is researched in detail from the configuration of the test field, the measurement and the calculation steps. This test procedure is in accordance with international standards. The test coordinates were performed with the Sokkia SET 520K electronic total station. The experimental results show that the experimental standard deviation of coordinates reaches $\pm 0.8 \mathrm{~mm}$. $\bigcirc$

\section{GIẢI PHÁP HIỆU CHỈNH TỌA ĐỘ VÀ ĐỘ CAO ĐIỂM.....}

(Tiếp theo trang 17)

[2]. Đinh Công Hòa (2010). Lập trình bài toán trắc địa cơ sở. Nhà xuất bản giao thông vận tải, 266-338.

\section{Summary}

Solution for correcting elevation and coordinate measured by GNSS/CORS Technology when combined with traditional measurement method in large scale mapping.

\section{Hoang Thi Thuy, Hanoi University of Mining and Geology, Vietnam}

In large-scale mapping, method of using electronic total stations (TS) can provide the most reliable results, especially in residential areas and vegetated areas. Meanwhile, GNSS/CORS technology has advantage of achieving fast results and it is very efficient in a well-ventilated area. In order to take advantage of these two methods, we propose the application of the coordinate and elevation adjustment problem to calibrate the map established by using GNSS/CORS technology on the map established by TS by using ground control points or clear feature points. In case of using GNSS/CORS for mapping, it is necessary to use TS to measure obscured points, these points can be measured in the assumed coordinate system, then use TS to measure some clear feature points which was determined by GNSS/CORS, these can be used as key points to match the area measured by TS to the area measured by GNSS/CORS. The result of the study is a programming module which can automatically correct the coordinate and elevation of the measured points when applying the combination of GNSS/CORS and TS for mapping. The experimental results showed that the proposed method can be applied for establishing topographic and cadastral maps from data derived by integrating GNSS/CORS and TS.O 